\title{
The Study of Y-aromaticity. IV. Tridentenes with irregular arm lengths
}

\section{OHMAE, Takayuki}

Department of Human Nutrition, Faculty of Nursing and Human Nutrition, Yamaguchi Prefectural University, 3-2-1 Sakurabatake, Yamaguchi City, Yamaguchi 753-8502 Japan

\begin{abstract}
To increase the number of synthetic targets having Y-aromaticity, we proposed $(n, l, m)$ tridentenes, in which the lengths of the three linear conjugated systems, extending from the central atom of the Y-shaped structure of n-tridentene were made irregular. The calculation results obtained using the semiempirical molecular orbital method suggested the existence of a compound that is energetically stabilized and 43 compounds with expectable kinetic stability.
\end{abstract}

Key Words: Y-aromaticity, Tridentene, HOMO-LUMO gap, MOPAC.

\section{Introduction}

The concept of Y-aromaticity was introduced to explain the strong basicity of guanidine [1]. This new concept of aromaticity, which does not premise on the existence of a cyclic $\pi$ electron conjugated system to be essential for the conventional aromaticity concept, has an attractive potential as a new working hypothesis for molecular design and till now, many studies have been conducted in this field [2-8]. However, since a rule corresponding to the $4 \mathrm{n}$ + 2 rule [9] in the conventional theory of aromaticity was unknown for Y-aromaticity, systematic studies have not been performed theoretically or experimentally.

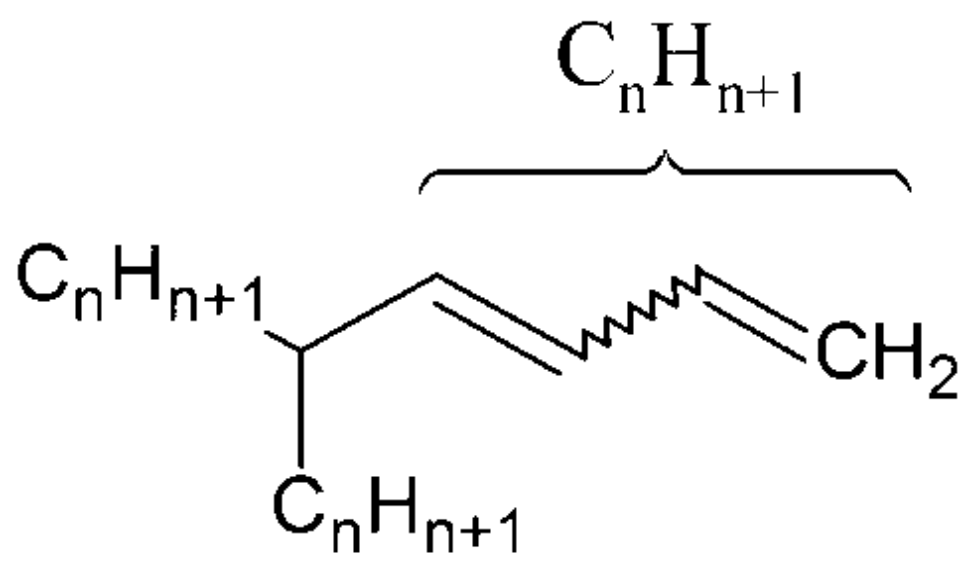

Figure 1. n-tridentene.

Therefore, the author proposed n-tridentene shown in Fig. 1 as an annulene-equivalent molecule in Y-aromaticity and derived the magic number in Y-aromaticity expressed by Eq. 1 [10]. Here it is assumed that the $\pi$-electron conjugated system in the structure forms a closed shell as a necessary condition for energetic stability. 


$$
\mathrm{N}=3 \mathrm{n}-\frac{1-(-1)^{\mathrm{n}}}{2}, \mathrm{n}=1,2,3, \cdots
$$

Furthermore, to examine the effectiveness of the obtained magic numbers, semiempirical molecular orbital calculations were performed [11], and two synthetic targets, typhoon tridentene and cyclone tridentene, shown in Fig. 2 were found, which are interesting also from a stereochemical point of view [12].

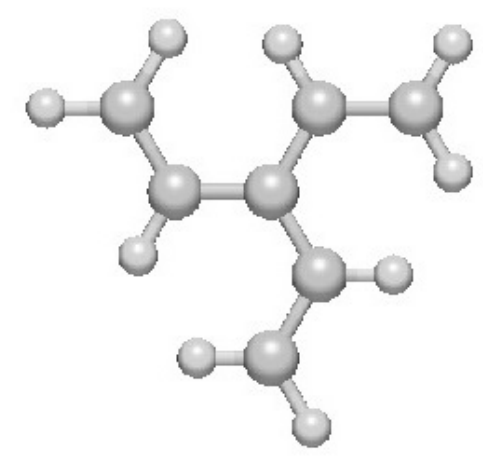

a

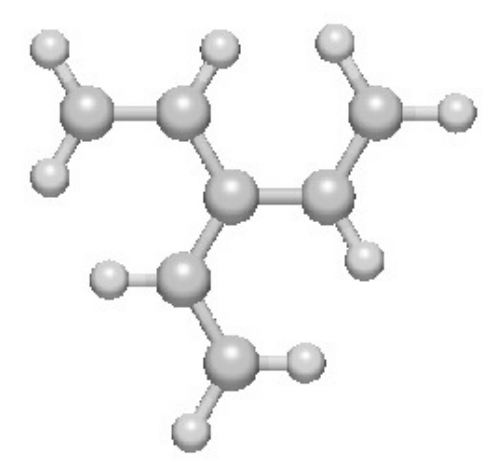

b

Figure 2. Typhoon tridentene and cyclone tridentene. $\mathrm{a}$ : typhoon tridentene, $\mathrm{b}$ : cyclone tridentene.

In this report, aiming to promote further progress of synthetic chemical research on $\mathrm{Y}$ shaped aromatic compounds, which are promising not only as novel aromatic compounds but also as basic structure of ionic fluids [13], we present our results of theoretical studies on the energetic and kinetic stability of a system in which the lengths of the three arms of $n$ tridentene are irregular, as illustrated in Fig.3. Henceforth, the molecule shown in Fig. 3 is referred to as $(n, l, m)$-tridentene.

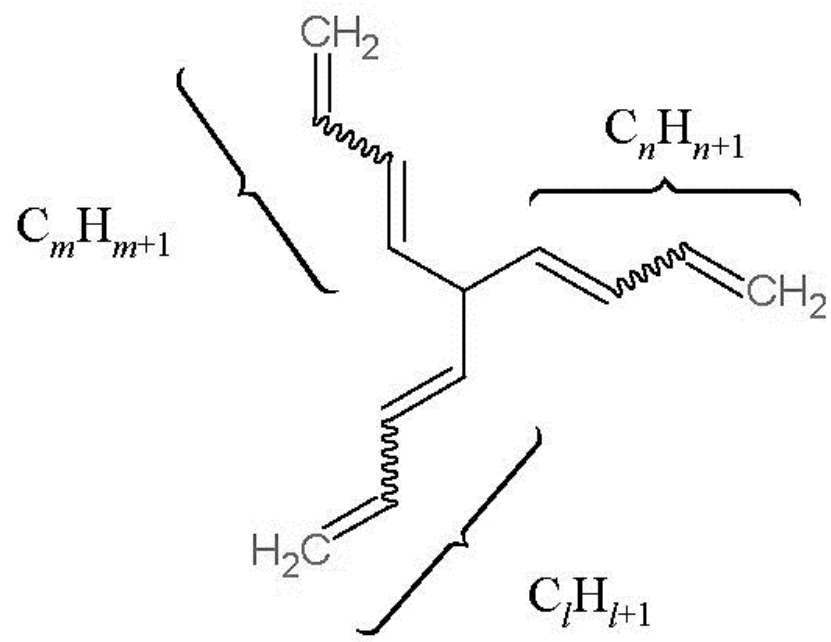

Figure 3. $(n, l, m)$-tridentene with radially arranged arms. 


\section{Calculation method and model compounds}

\subsection{Calculation method}

The structural optimization calculation in the ground state of model compounds was performed using the PM5 method [14,15] of SCIGRESS MO Compact ver.1.0.0 Standard (Fujitsu), and the heat of formation, MO data, and the optimized structure were determined.

\subsection{Model compounds}

As model compounds, $(n, l, m)$-tridentenes shown in Fig. 3 were adopted. Here, the values of $n, l$, and $m$ were each in the range of $1-6$, and when all of $n, l$, and $m$ had the same value, they were not included in the calculation. Additionally, the calculation target was limited to the minimum charged state in which the $\pi$-electron conjugated system of the respective systems can be expected to form a closed shell.

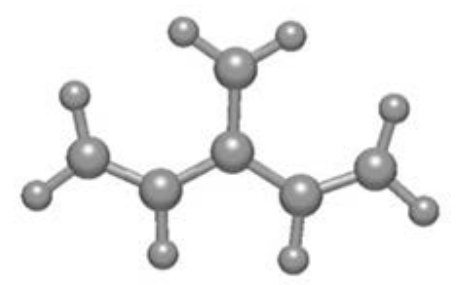

\section{a}

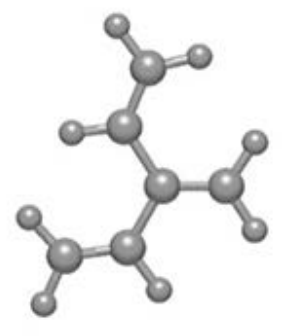

b

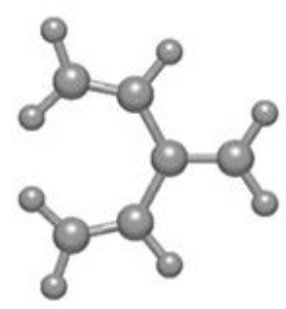

C

Figure 4. (1, 2, 2)-tridentene arm configuration.

$\mathrm{a}$ : symmetrical open configuration, $\mathrm{b}$ : radial configuration, $\mathrm{c}:$ symmetrical compact configuration.

As shown in Section 5, Appendix, the system to be calculated was selected within the range of Hückel approximation. Therefore, the arm arrangement, when performing semiempirical molecular orbital calculations is, in principle, a planar arrangement with radially open arms of tridentene, which is expected to minimize spatial interactions that are not considered in the Hückel approximation. However, as shown in Table 1, preliminary calculation results suggest that $(1, m, m)$-tridentene becomes more stable when two arms consisting of $m$ carbon atoms are arranged symmetrically about the arm of length 1 , rather than being arranged radially. 
Thus, the arrangement of the arms of $(1, m, m)$-tridentene was calculated in the symmetrical and open plane configuration shown in Fig.4.

Table 1. Heat of formation $\left(\Delta \mathrm{H}_{\mathrm{f}}\right)$ of $(1,2,2)$-tridentene and effect of arm configuration on HOMO-LUMO gap $\left(\Delta \varepsilon_{\mathrm{HL}}\right)$.

\begin{tabular}{|l|l|l|}
\hline Arm configuration & $\Delta \mathrm{H}_{\mathrm{f}}(\mathrm{kcal} / \mathrm{mol})$ & $\Delta \varepsilon_{\mathrm{HL}}(\mathrm{eV})$ \\
\hline \hline symmetrical open configuration & 44.81911 & 9.3524 \\
\hline radial configuration & 45.37969 & 9.3226 \\
\hline symmetrical compact configuration & 48.59780 & 9.2698 \\
\hline
\end{tabular}

\section{Results and discussion}

For the 82 types of $(n, l, m)$-tridentene $(n, l, m=1-6)$, in which the $\pi$-electron conjugated system can be expected to form a closed shell within the scope of Hückel approximation, the calculation results of the heat of formation and HOMO-LUMO gap are summarized in Table 2. Furthermore, Table 2 also shows the calculation results of 31 types of linear conjugated systems $\left(\mathrm{LN}^{ \pm \mathrm{q}}\right)$ consisting of the same number of carbon atoms and $\pi$ electrons as each tridentene as the reference standards for energetic stabilization [10, 11]. Note that the selection of the charge state of tridentenes to be calculated was according to Table 4, which was obtained as a result discussed in Section 5.

Comparing the heat of formation values, only the divalent cation of $(1,1,3)$-tridentene with a scorpion-shaped arm configuration could be expected to possess energetic stability. Furthermore, as this ion has a HOMO-LUMO gap larger than the corresponding reference system, it is a promising synthetic target that can also exhibit kinetic stability.

Next, based on the examination of the HOMO-LUMO gap values, the 43 types of tridentenes were suggested as shown in Table 3, including $(1,1,3)$-tridentene, which may have kinetic stability. As shown in Table 3, all the ions expected to be kinetically stabilized, were paired except for $(2,3,5)$-tridentene. As the calculation results in Table 2 suggest, the HOMO-LUMO gap of the anion tends to be smaller than that of the corresponding cation. The difference in the value of the HOMO-LUMO gap in the reference system was small for $(2,3,5)$-tridentene cation and in the anion this difference is the reversed. Further, the value of the HOMO-LUMO gap of tridentenes having $(n, l, m)$ parity of (odd, odd, odd) and (even, even, even) is, without exception, greater than the value of the HOMO-LUMO gap of the reference system, suggesting kinetic stability. This result is not conflicting with the previously reported calculation results of n-tridentene [11]. 
Table 2. Heat of formation and HOMO-LUMO gap of $(n, l, m)$-tridentene and its reference system calculated using the semiempirical molecular orbital method.

\begin{tabular}{|c|c|c|c|c|c|}
\hline Species & $\Delta \mathrm{H}_{\mathrm{f}}$ & $\Delta \varepsilon_{\mathrm{HL}}$ & Species & $\Delta \mathrm{H}_{\mathrm{f}}$ & $\Delta \varepsilon_{\mathrm{HL}}$ \\
\hline $\mathrm{L}^{+}$ & 220.19442 & 8.2412 & L12 & 82.68174 & 7.6336 \\
\hline$(1,1,2)^{+}$ & 237.50965 & 6.9420 & $(3,4,4)$ & 88.01632 & 7.7553 \\
\hline $\mathrm{L}^{-}$ & 23.05440 & 7.9245 & $(1,4,6)$ & 86.41614 & 8.0987 \\
\hline$(1,1,2)^{-}$ & 39.77129 & 6.7508 & $(2,3,6)$ & 87.69566 & 7.7355 \\
\hline L6 & 42.26763 & 8.8868 & $(2,4,5)$ & 87.71962 & 7.6045 \\
\hline$(1,2,2)$ & 44.81911 & 9.3524 & $\mathrm{~L} 12^{2+}$ & 491.25597 & 6.5568 \\
\hline $\mathrm{L}^{2+}$ & 530.93140 & 8.0193 & $(1,5,5)^{2+}$ & 497.51106 & 7.2115 \\
\hline$(1,1,3)^{2+}$ & 529.92260 & 8.7783 & $(3,3,5)^{2+}$ & 504.41535 & 6.9909 \\
\hline $\mathrm{L}^{2-}$ & 133.75324 & 7.6103 & $\mathrm{~L} 12^{2-}$ & 92.99543 & 6.4635 \\
\hline$(1,1,3)^{2-}$ & 134.20527 & 8.4565 & $(1,5,5)^{2-}$ & 98.67694 & 7.0749 \\
\hline $\mathrm{L}^{+}$ & 223.98636 & 7.2893 & $(3,3,5)^{2-}$ & 105.59604 & 6.8657 \\
\hline$(1,1,4)^{+}$ & 249.72233 & 5.4701 & $\mathrm{~L} 13^{+}$ & 251.32290 & 5.9174 \\
\hline$(1,2,3)^{+}$ & 236.53387 & 6.7852 & $(2,5,5)^{+}$ & 259.59853 & 5.9619 \\
\hline $\mathrm{L}^{-}$ & 26.37724 & 7.1055 & $(3,3,6)^{+}$ & 268.77878 & 4.9486 \\
\hline$(1,1,4)^{-}$ & 50.53314 & 5.4208 & $(1,5,6)^{+}$ & 267.21433 & 4.8117 \\
\hline$(1,2,3)^{-}$ & 38.68867 & 6.6002 & $(2,4,6)^{+}$ & 254.05241 & 6.3410 \\
\hline L8 & 55.74715 & 8.2757 & $(3,4,5)^{+}$ & 263.50234 & 5.5903 \\
\hline$(2,2,3)$ & 60.49113 & 8.3565 & $\mathrm{~L}^{-} 3^{-}$ & 52.64671 & 5.9037 \\
\hline$(1,2,4)$ & 59.18427 & 8.6932 & $(2,5,5)^{-}$ & 60.79540 & 5.9145 \\
\hline $\mathrm{L}^{2+}$ & 508.62159 & 7.3354 & $(3,3,6)^{-}$ & 69.20089 & 4.9290 \\
\hline$(1,3,3)^{2+}$ & 512.92679 & 8.2349 & $(1,5,6)^{-}$ & 67.56204 & 4.8121 \\
\hline$(1,1,5)^{2+}$ & 514.35880 & 7.4584 & $(2,4,6)^{-}$ & 54.90981 & 6.2753 \\
\hline $\mathrm{L}^{2-}$ & 110.92388 & 7.0920 & $(3,4,5)^{-}$ & 64.10424 & 5.5559 \\
\hline$(1,3,3)^{2-}$ & 115.36273 & 7.9866 & L14 & 96.14792 & 7.4536 \\
\hline$(1,1,5)^{2-}$ & 116.96537 & 7.3321 & $(1,6,6)$ & 99.24572 & 7.9647 \\
\hline $\mathrm{L}^{+}$ & 231.32130 & 6.6683 & $(4,4,5)$ & 101.44397 & 7.4153 \\
\hline$(2,3,3)^{+}$ & 242.68367 & 6.5443 & $(2,5,6)$ & 101.13134 & 7.4383 \\
\hline$(2,2,4)^{+}$ & 234.69826 & 7.2593 & $(3,4,6)$ & 101.51474 & 7.5750 \\
\hline$(1,1,6)^{+}$ & 262.06822 & 4.5620 & $\mathrm{~L}_{14}{ }^{2+}$ & 490.03617 & 6.3152 \\
\hline$(1,2,5)^{+}$ & 240.71501 & 6.5262 & $(3,5,5)^{2+}$ & 504.22082 & 6.5816 \\
\hline$(1,3,4)^{+}$ & 249.61170 & 5.5802 & $\mathrm{~L} 14^{2-}$ & 91.57841 & 6.2588 \\
\hline $\mathrm{L}^{-}$ & 33.30962 & 6.5656 & $(3,5,5)^{2-}$ & 105.00543 & 6.4893 \\
\hline$(2,3,3)^{-}$ & 44.48969 & 6.4064 & $\mathrm{~L}_{1} 5^{+}$ & 262.80748 & 5.6749 \\
\hline$(2,2,4)^{-}$ & 36.21446 & 7.0836 & $(4,4,6)^{+}$ & 264.51579 & 6.2934 \\
\hline$(1,1,6)^{-}$ & 62.31878 & 4.5644 & $(2,6,6)^{+}$ & 265.15938 & 6.0785 \\
\hline$(1,2,5)^{-}$ & 42.47738 & 6.3794 & $(4,5,5)^{+}$ & 273.11376 & 5.5309 \\
\hline$(1,3,4)^{-}$ & 50.39352 & 5.5182 & $(3,5,6)^{+}$ & 276.70661 & 4.9990 \\
\hline L10 & 69.21545 & 7.8910 & $\mathrm{~L}^{1} 5^{-}$ & 63.85322 & 5.6857 \\
\hline$(1,4,4)$ & 72.24859 & 8.4440 & $(4,4,6)^{-}$ & 65.10180 & 6.2221 \\
\hline$(2,2,5)$ & 73.93630 & 7.8630 & $(2,6,6)^{-}$ & 65.72953 & 6.0499 \\
\hline$(1,2,6)$ & 72.68799 & 8.2084 & $(4,5,5)^{-}$ & 73.51516 & 5.5196 \\
\hline
\end{tabular}




\begin{tabular}{|l|r|r||r|r|r|}
\hline$(2,3,4)$ & 74.20812 & 7.9949 & $(3,5,6)^{-}$ & 77.01125 & 4.9851 \\
\hline $\mathrm{L} 10^{2+}$ & 496.74026 & 6.8789 & $\mathrm{~L} 16$ & 109.61430 & 7.3227 \\
\hline$(1,3,5)^{2+}$ & 504.31713 & 7.4483 & $(3,6,6)$ & 115.00717 & 7.4511 \\
\hline $\mathrm{L}^{2-}$ & 98.71819 & 6.7291 & $(4,5,6)$ & 114.93372 & 7.2881 \\
\hline$(1,3,5)^{2-}$ & 106.14551 & 7.3200 & $\mathrm{~L}^{2} 7^{+}$ & 274.87314 & 5.4820 \\
\hline${\mathrm{L} 11^{+}}^{+}$ & 240.68468 & 6.2350 & $(4,6,6)^{+}$ & 275.97906 & 6.1137 \\
\hline$(2,4,4)^{+}$ & 243.62841 & 6.8643 & $(5,5,6)^{+}$ & 286.24837 & 5.0490 \\
\hline$(3,3,4)^{+}$ & 255.87427 & 5.6584 & $\mathrm{~L}^{+}$ & 75.67003 & 5.5095 \\
\hline$(2,2,6)^{+}$ & 244.00541 & 6.5555 & $(4,6,6)^{-}$ & 76.30622 & 6.0716 \\
\hline$(1,3,6)^{+}$ & 262.40873 & 4.7476 & $(5,5,6)^{-}$ & 86.35228 & 5.0503 \\
\hline$(1,4,5)^{+}$ & 253.81457 & 5.5477 & $\mathrm{~L} 18$ & 123.08088 & 7.2249 \\
\hline$(2,3,5)^{+}$ & 250.18062 & 6.2481 & $(5,6,6)$ & 128.43153 & 7.1874 \\
\hline L11 & 42.32035 & 6.1852 & & & \\
\hline$(2,4,4)^{-}$ & 44.78756 & 6.7429 & & & \\
\hline$(3,3,4)^{-}$ & 56.67921 & 5.5965 & & & \\
\hline$(2,2,6)^{-}$ & 45.19778 & 6.4618 & & & \\
\hline$(1,3,6)^{-}$ & 62.70861 & 4.7453 & & & \\
\hline$(1,4,5)^{-}$ & 54.80369 & 5.4890 & & & \\
\hline$(2,3,5)^{-}$ & 51.73304 & 6.1515 & & & \\
\hline
\end{tabular}

$\mathrm{LN}^{\mathrm{p}}$ : p-valent ion of reference system constituted by $\mathrm{N}$ atoms.

$(n, l, m)^{\mathrm{q}}:$ q-valent ion of $(n, l, m)$-tridentene.

$\Delta \mathrm{H}_{\mathrm{f}}$ : heat of formation, kcal/mol. $\Delta \varepsilon_{\mathrm{HL}}:$ HOMO-LUMO gap, eV.

Table 3. ( $n, l, m)$-tridentenes expected to be kinetically stable.

\begin{tabular}{|l|l|}
\hline Charge state & $(n, l, m)$-tridentene \\
\hline \hline $2+$ & $(1,1,3),(1,3,3),(1,1,5),(1,3,5),(1,5,5)$, \\
& $(3,3,5),(3,5,5)$ \\
\hline+ & $(2,2,4),(2,4,4),(2,2,6),(2,3,5),(2,5,5)$, \\
& $(2,4,6),(4,4,6),(2,6,6),(4,6,6)$ \\
\hline 0 & $(1,2,2),(2,2,3),(1,2,4),(1,4,4),(1,2,6)$, \\
& $(2,3,4),(3,4,4),(1,4,6),(2,3,6),(1,6,6)$, \\
& $(3,4,6),(3,6,6)$ \\
\hline- & $(2,2,4),(2,4,4),(2,2,6),(2,5,5),(2,4,6)$, \\
& $(4,4,6),(2,6,6),(4,6,6)$ \\
\hline $2-$ & $(1,1,3),(1,3,3),(1,1,5),(1,3,5),(1,5,5)$, \\
& $(3,3,5),(3,5,5)$ \\
\hline
\end{tabular}




\section{Conclusion}

From the above results, it was concluded that the divalent cation of $(1,1,3)$-tridentene is a promising synthetic target. Further, from the viewpoint of kinetic stability, the 43 types of tridentenes, including $(1,1,3)$-tridentene, are also found to be worth considering as candidates for synthetic targets.

\section{Appendix}

The Hückel molecular orbital energy of $(n, l, m)$-tridentene shown in Fig. 3 is given by the pole of the Hückel approximation propagator G(z) at the central atom of the Y-shaped structure, expressed by Equation 2 [10].

$$
\mathrm{G}(\mathrm{z})^{-1}=\frac{1}{\mathrm{p}_{n} \mathrm{p}_{l} \mathrm{p}_{m}} \times\left\{\mathrm{zp}_{n} \mathrm{p}_{l} \mathrm{p}_{m}-\mathrm{p}_{n-1} \mathrm{p}_{l} \mathrm{p}_{m}-\mathrm{p}_{n} \mathrm{p}_{l-1} \mathrm{p}_{m}-\mathrm{p}_{n} \mathrm{p}_{l} \mathrm{p}_{m-1}\right\}
$$

Here, $\mathrm{p}_{n}$ is the Hückel determinant of a linear conjugated system and is generally given by Equation 3 [16].

$$
\mathrm{p}_{n}=\sum_{\mathrm{k}=0}^{\left[\frac{n}{2}\right]}-1^{\mathrm{k}}\left(\begin{array}{c}
n-1 \\
\mathrm{k}
\end{array}\right) \mathrm{z}^{n-2 \mathrm{k}}
$$

Here, $[n / 2]$ represents an integer that does not exceed $n / 2$. As is clear from Equation 3 , the lowest order of $\mathrm{p}_{n}$ is $\mathrm{z}$ to the 1st power when $\mathrm{n}$ is an odd number, and $\mathrm{z}$ to the 0th power when $\mathrm{n}$ is an even number. Considering this fact, it is easily possible to know the order of $\mathrm{z}$ that can be factored out of the braces in Equation 2.

Since the order of $\mathrm{z}$ that can be factored out of the braces corresponds to the number of socalled nonbonding orbitals, the qualitative distribution of Hückel molecular orbital energy of $(n, l, m)$-tridentene can be predicted by the combination of $(n, l, m)$ parity. For example, if ( $n$, $l, m)$ are all odd numbers, the lowest order of $\mathrm{p}_{n}, \mathrm{p}_{l}, \mathrm{p}_{m}$ is the 1 st order of $\mathrm{z}$, respectively. Therefore, the square of $\mathrm{z}$ is factored out of the braces of Equation 2. This means that when ( $n$, $l, m)$ are all odds, according to the Hückel molecular orbital theory, $(n+l+m-1) / 2$ bonding orbitals and $(n+l+m-1) / 2$ antibonding orbitals that sandwich the doubly degenerate nonbonding orbitals are formed in pairs, as shown in Fig. 5a. Here, the fact that $(n, l, m)$ tridentene is an alternant hydrocarbon was taken into consideration [17]. Furthermore, the neutral $(n, l, m)$-tridentene has $(n+l+m+1) \pi$ electrons. Therefore, as shown in Fig. 5a, the bonding orbitals of this molecule are completely occupied, and the doubly degenerated nonbonding orbitals are each semi-occupied.

Similarly, the distribution and occupied state of the Hückel molecular orbital shown in Fig. 5 can be obtained by considering the case according to the parity of $(n, l, m)$. 

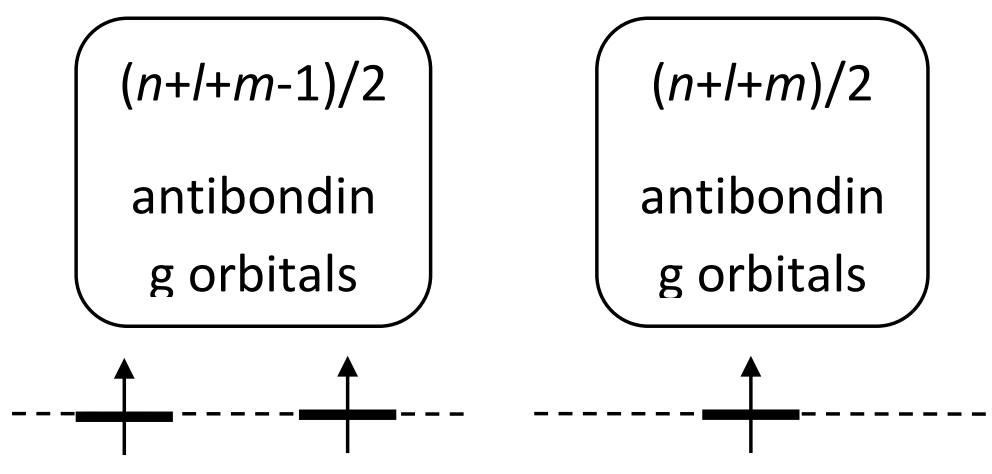

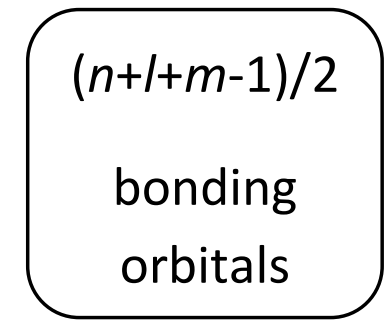

a

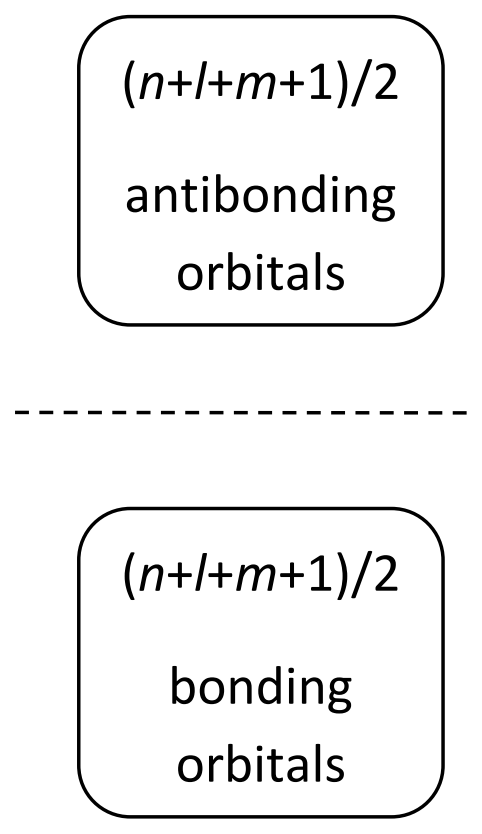

C

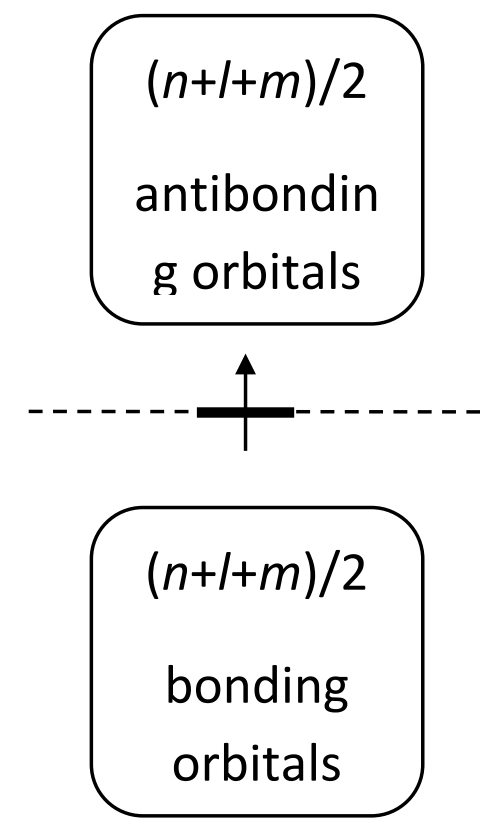

$\mathrm{d}$

Figure 5. $(n, l, m)$ parity and distribution of Hückel molecular orbitals.

a: $(n, l, m)=($ odd, odd, odd $), \mathrm{b}:(n, l, m)=($ odd, odd, even $)$,

c: $(n, l, m)=($ odd, even, even $), \mathrm{d}:(n, l, m)=($ even, even, even $)$. 
Here, having in mind that the purpose of this report was to enhance the synthetic chemical research of Y-aromatic compounds, systems in which the $\pi$-electron system forms a closed shell in a neutral or minimally charged state were selected as the calculation target and the results shown in Table 4 were obtained. It should be noted that even if the nonbonding orbital is completely occupied, the shell is taken as closed.

Table 4. $n, l, m$ parity and charge state of closed shell.

\begin{tabular}{|l|l|l|l|}
\hline$n$ parity & $l$ parity & $m$ parity & closed shell charge state \\
\hline \hline odd & odd & odd & \pm 2 \\
\hline odd & odd & even & \pm 1 \\
\hline odd & even & even & 0 \\
\hline even & even & even & \pm 1 \\
\hline
\end{tabular}

The author thanks Crimson Interactive Pvt. Ltd. (Ulatus) - www.ulatus.jp for their assistance in manuscript translation.

\section{References and notes}

[1] P. Gund., J. Chem. Educ., 49, 100-103(1972).

[2] L. Grajcar, G. Berthier, J. Faure, J.-P. Fleury, Theor. Chim. Acta, 71, 299-304(1987).

[3] T. Ohwada, H. Kagawa, H. Ichikawa, Bull. Chem. Soc. Jpn., 1977, 70, 2411-2415.

[4] T. P. Radhakrishnan, I. Agranat, J. Org. Chem., 2001, 66, 3215-3219.

[5] A. Dworkin, R. Naumann, C. Seigfred, J. M. Karty, Y. Mo, J. Org. Chem., 2005, 70, 7605-7616.

[6] I. J. Binoy, C. James, J. I. Hubert, V. S. Jayakumar, J. Mol. Struct., 2006, 784, 32-46.

[7] I. Rozas, G. S. Sanz, I. Alkarta, J. Elguero, J. Phys. Org. Chem., 2013, 26, 378-385.

[8] E. Kleipeter, A. Koch, Tetrahedron, 2016, 72, 1675-1685.

[9] M. Sainsbury, Aromatic Chemistry, Oxford University Press., New York, 1992, pp.3-7.

[10] T. Ohmae, Journal of Computer Aided Chemistry, 2018, 19, 19-25. This report shall be the study of Y-aromaticity I.

[11]T. Ohmae, Journal of Computer Aided Chemistry, 2019, 20, 18-22. This report shall be the study of Y-aromaticity II.

[12] T. Ohmae, Bulletin of the Faculty of Nursing and Nutrition Yamaguchi Prefectural University, 2020, 13, 55-62. This report shall be the study of Y-aromaticity III.

[13] T. Takamuku, Newtron network news, 2019, 29, 95-99.

[14] J. J. Stewart, J. Comput. Chem., 1989, 10, 221-264.

[15] J. J. Stewart, MOPAC2002 manual, Fujitsu Limited, Tokyo, 2001.

[16] S. Aono, K. Nishikawa, Bull. Chem. Soc. Jpn., 1980, 53, 3418-3423.

[17]I. N. Levine, Quantum Chemistry, Person Education, London, 2009, p.650. 